\title{
PINK1/Parkin-mediated mitophagy plays a protective role in the bone impairment caused by aluminum exposure
}

Yilong Cui, Miao Song, Bonan Xiao, Wanyue Huang, Jian Zhang, Xuliang Zhang, Bing Shao, Yanfei Han, Yanfei $\mathrm{Li}^{* 1}$

Key Laboratory of the Provincial Education, Department of Heilongjiang for Common Animal

Disease Prevention and Treatment, College of Veterinary Medicine, Northeast Agricultural University, Harbin 150030, China.

*Corresponding author at: College of Veterinary Medicine, Northeast Agricultural University, NO. 600, Changjiang Road, Harbin 150030, China.

E-mail address: liyanfei@neau.edu.cn (Y.F. Li); Tel.: +86 13936574268; fax: +86 451 55191672 . 
Table S1

The mortality of acute toxicity tests in C57 mice.

\begin{tabular}{ccccc}
\hline Groups & $\mathrm{AlCl}_{3}$ Doses $(\mathrm{mg} / \mathrm{kg})$ & Number of samples & Deaths & Mortality rate $(100 \%)$ \\
\hline I & 2805.0 & 8 & 8 & 100 \\
II & 2384.3 & 8 & 7 & 87.5 \\
III & 2026.6 & 8 & 7 & 87.5 \\
IV & 1722.6 & 8 & 2 & 25 \\
V & 1464.2 & 8 & 2 & 25 \\
VI & 1244.5 & 8 & 0 & 0 \\
VII & 0 & 8 & 0 & 0 \\
\hline
\end{tabular}

The median lethal dose $\left(\mathrm{LD}_{50}\right)$ is $1793.64 \mathrm{mg} / \mathrm{kg}$ calculated by SPSS 22.0 software (SPSS Incorporated, USA). The $95 \%$ confidence limit of $\mathrm{LD}_{50}$ is $1615.77 \sim 1985.77 \mathrm{mg} / \mathrm{kg}$. The $95 \%$ mean confidence limit of the $\mathrm{LD}_{50}$ is $1793.64 \pm 185 \mathrm{mg} / \mathrm{kg}$. Mice were obtained from Liaoning Changsheng Biotech Co., Ltd. (NO.C-HLJ2019080543, Benxi, China). 
Table S2

Primer sequences and amplification lengths of destination fragments.

\begin{tabular}{|c|c|c|c|c|}
\hline Genes & Gene numbers & Primer sequences & $\begin{array}{c}\text { Primer } \\
\text { lengths (bp) }\end{array}$ & $\begin{array}{c}\text { Product } \\
\text { lengths (bp) }\end{array}$ \\
\hline \multirow{2}{*}{ p62 } & \multirow{2}{*}{ NM_001290769.1 } & F:5' CCCATCTACAGAGGCTGATCC 3' & 21 & \multirow{2}{*}{184} \\
\hline & & R:5' TTGGCCACAGCACTATCACA 3' & 20 & \\
\hline \multirow{2}{*}{$\mathrm{LC} 3$} & \multirow{2}{*}{ NM_026160.5 } & F:5' GAACCGCAGACGCATCTCT 3' & 19 & \multirow{2}{*}{171} \\
\hline & & R:5' TGATCACCGGGATCTTACTGG 3' & 21 & \\
\hline \multirow{2}{*}{ PINK1 } & \multirow{2}{*}{ NM_026880.2 } & F:5' GCAGCAGTCAGCAGCCACTC 3' & 20 & \multirow{2}{*}{103} \\
\hline & & R:5' AGCCTCACACTCCAGGTTAGCC 3' & 22 & \\
\hline \multirow{2}{*}{ Parkin } & \multirow{2}{*}{ NM_001317726.1 } & F:5' AAGGAAGTGGTTGCTAAGCGACAG 3' & 24 & \multirow{2}{*}{156} \\
\hline & & R:5' ATGACTTCTCCTCCGTGGTCTCTG 3' & 24 & \\
\hline \multirow{2}{*}{ BGP } & \multirow{2}{*}{ NM_007541.3 } & F:5' ACCCTGGCTGCGCTCTGTC 3' & 19 & \multirow{2}{*}{149} \\
\hline & & R:5' GGATCTGGGCTGGGGACTGAG 3' & 21 & \\
\hline \multirow{2}{*}{ B-ALP } & \multirow{2}{*}{ NM_001287172.1 } & F:5' TCATTCCCACGTTTTCACATTC 3' & 22 & \multirow{2}{*}{187} \\
\hline & & R:5' GTTGTTGTGAGCGTAATCTACC 3' & 22 & \\
\hline \multirow{2}{*}{ Col- I } & \multirow{2}{*}{ NM_007742.4 } & F:5' TGAACGTGGTGTACAAGGTC 3' & 20 & \multirow{2}{*}{234} \\
\hline & & R:5' CCATCTTTACCAGGAGAACCAT 3' & 22 & \\
\hline \multirow{2}{*}{$\beta$-actin } & \multirow{2}{*}{ NM_007393.5 } & F:5' GTTGGAGCAAACATCCCCCA 3' & 20 & \multirow{2}{*}{187} \\
\hline & & R:5' ACGCGACCATCCTCCTCTTA 3' & 20 & \\
\hline
\end{tabular}




\section{Selection of $\mathrm{AlCl}_{3}$ dose in cell test}

As shown in Fig. S1, compared with the $0 \mathrm{mM}$ group, the protein expressions of PINK1, Parkin and LC3 I / I were significantly increased with the increase of the exposure concentration of $\mathrm{AlCl}_{3}$, while the protein expression of p62 was significantly decreased $(P<0.01)$. In the $2 \mathrm{mM} \mathrm{AlCl}_{3}$ treatment group, the expressions of mitophagy-related proteins were the highest, indicating that mitophagy was the most obvious, so we chose this dose for the next experiment.
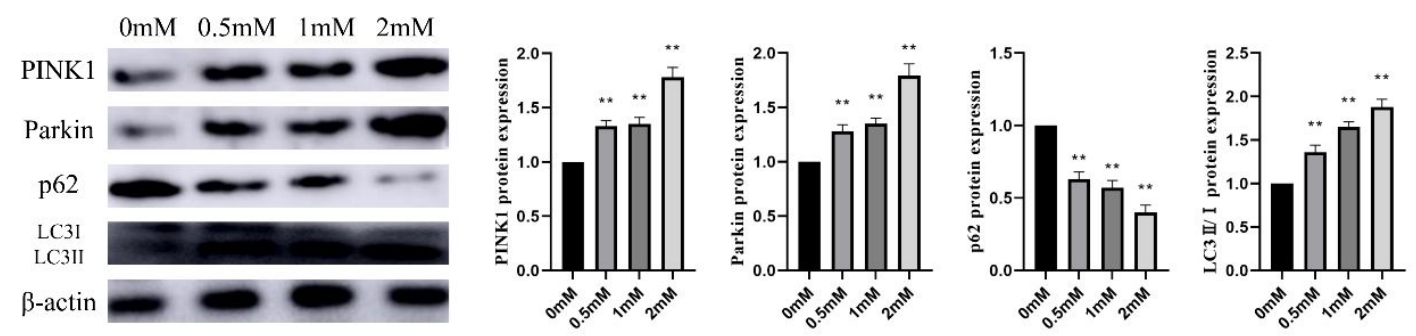

Fig. S1. The effect of $0 \mathrm{mM}, 0.5 \mathrm{mM}, 1 \mathrm{mM}$ and $2 \mathrm{mM} \mathrm{AlCl}_{3}$ on the mitophagy of MC3T3-E1 cells for $24 \mathrm{~h}(\mathrm{n}=3)$. All data were expressed as means $\pm \mathrm{SD} . * P<0.05$ and $* * P<0.01$ versus the $0 \mathrm{mM}$. 